\title{
Transitions from phase-locked dynamics to chaos in a piecewise-linear map
}

\section{Zhusubaliyev, Z.T.; Mosekilde, Erik; De, S.; Banerjee, S.}

\section{Published in:}

Physical Review E

Link to article, DOI:

10.1103/PhysRevE.77.026206

Publication date:

2008

Document Version

Publisher's PDF, also known as Version of record

Link back to DTU Orbit

\section{Citation (APA):}

Zhusubaliyev, Z. T., Mosekilde, E., De, S., \& Banerjee, S. (2008). Transitions from phase-locked dynamics to chaos in a piecewise-linear map. Physical Review E, 77(2), 026206.

https://doi.org/10.1103/PhysRevE.77.026206

\section{General rights}

Copyright and moral rights for the publications made accessible in the public portal are retained by the authors and/or other copyright owners and it is a condition of accessing publications that users recognise and abide by the legal requirements associated with these rights.

- Users may download and print one copy of any publication from the public portal for the purpose of private study or research.

- You may not further distribute the material or use it for any profit-making activity or commercial gain

- You may freely distribute the URL identifying the publication in the public portal

If you believe that this document breaches copyright please contact us providing details, and we will remove access to the work immediately and investigate your claim 


\title{
Transitions from phase-locked dynamics to chaos in a piecewise-linear map
}

\author{
Zhanybai T. Zhusubaliyev* \\ Kursk State Technical University, Dept. of Computer Science, 50 Years of October Street, 94, Kursk 305040, Russia \\ Erik Mosekilde ${ }^{\dagger}$ \\ Complex Systems Group, Department of Physics, The Technical University of Denmark, 2800 Lyngby, Denmark \\ Soma De $\mathrm{D}^{\ddagger}$ \\ Department of Mathematics and Centre for Theoretical Studies, Indian Institute of Technology, Kharagpur-721302, India \\ Soumitro Banerjee ${ }^{\S}$ \\ Department of Electrical Engineering and Centre for Theoretical Studies, Indian Institute of Technology, Kharagpur-721302, India
}

(Received 27 October 2007; published 11 February 2008)

\begin{abstract}
Recent work has shown that torus formation in piecewise-smooth maps can take place through a special type of border-collision bifurcation in which a pair of complex conjugate multipliers for a stable cycle abruptly jump out of the unit circle. Transitions from an ergodic to a resonant torus take place via border-collision fold bifurcations. We examine the transition to chaos through torus destruction in such maps. Considering a piecewise-linear normal-form map we show that this transition, by virtue of the interplay of border-collision bifurcations with period-doubling and homoclinic bifurcations, can involve mechanisms that differ qualitatively from those described by Afraimovich and Shilnikov.
\end{abstract}

DOI: 10.1103/PhysRevE.77.026206

PACS number(s): 05.45.Gg, 05.45.Pq

\section{INTRODUCTION}

Many problems in engineering and applied science lead us to consider piecewise-smooth maps. Examples of such systems include relay and pulse-width modulated control systems $[1,2]$, mechanical systems with dry friction or impacts [3], and managerial or economic systems with welldefined intervention thresholds [4].

As a parameter is varied, the fixed point for the Poincare map of such a system may move in phase space and collide with the boundary between two smooth regions. When this happens, the Jacobian matrix can change abruptly, leading to a special class of nonlinear dynamic phenomena known as border-collision bifurcations [5-9].

A simple type of border-collision bifurcation consists in the direct transition from one periodic orbit into another with the same period. However, more complicated phenomena are also possible, including period-multiplying bifurcations, multiple-choice bifurcations, and direct transition from periodicity to chaos [2,10-15]. Border-collision-related bifurcations also include corner-collision, sliding, and grazing bifurcations [16-18].

Piecewise-smooth systems can also display quasiperiodic behavior. In a series of recent publications [19-22] we have shown that border-collision bifurcations can lead to the birth of an invariant torus associated with quasiperiodic or phaselocked periodic dynamics. This transition resembles the wellknown Neimark-Sacker bifurcation in several respects. How-

\footnotetext{
*zhanybai@mail.kursk.ru

†Erik.Mosekilde@fysik.dtu.dk

†soma@cts.iitkgp.ernet.in

§soumitro@ee.iitkgp.ernet.in
}

ever, rather than through a continuous crossing of a pair of complex-conjugate multipliers of the periodic orbit through the unit circle, the border-collision bifurcation involves a jump of the multipliers from the inside to the outside of this circle. We have also demonstrated the existence of a special type of border-collision bifurcation in which a stable periodic orbit arises simultaneously with a quasiperiodic or phaselocked invariant torus $[19,23]$.

Along with the period-doubling route and various types of intermittency transitions, the formation and subsequent destruction of a two-dimensional torus is one of the classic routes to chaos in dissipative systems. Before breakdown, the resonance torus typically loses its smoothness in discrete points through folding (or winding) of the involved manifolds, and this loss of smoothness then spreads to the entire torus surface through local (i.e., saddle-node) or global (i.e., homoclinic or heteroclinic) bifurcations.

The basic theorem for the destruction of a twodimensional torus in smooth dynamical systems was proved by Afraimovich and Shilnikov [24], and three possible routes for the appearance of chaotic dynamics were described. The generic character of these processes has since been confirmed numerically as well as experimentally for wide classes of both continuous- and discrete-time systems [25-27].

The purpose of the present paper is to investigate some of the mechanisms that are involved in the transitions from phase-locked periodic dynamics to chaos in nonsmooth maps. With this purpose we follow the bifurcations that take place as the point of operation for a piecewise-linear normalform map leaves the 1:4 resonance tongue along three different routes. We show that the interplay between perioddoubling, border-collision, and homoclinic bifurcations leads to transitions that are qualitatively different from those of Afraimovich and Shilnikov. In particular, we consider a route 
in which a homoclinic bifurcation first destroys the resonance torus while leaving the original stable-node cycle. This node subsequently undergoes a period-doubling bifurcation combined with a simultaneous border-collision bifurcation for the appearing subharmonic, and chaos arises. Other routes involve regions of coexistence of periodic and chaotic oscillations or of different chaotic oscillations. The paper discusses the specific features of these routes and outlines some of the characteristic differences between the routes followed in smooth and nonsmooth maps.

\section{PIECEWISE-LINEAR NORMAL-FORM MAP}

It is well known that dynamical phenomena related to border-collision bifurcations can be examined by means of a piecewise-linear approximation to the Poincare map in the neighborhood of the border-crossing fixed point, expressed in the convenient normal form $[6,7,10]$

$$
F:\left(\begin{array}{l}
x \\
y
\end{array}\right) \mapsto \begin{cases}F_{1}(x, y), & x \leqslant 0, \\
F_{2}(x, y), & x \geqslant 0,\end{cases}
$$

where

$$
\begin{gathered}
F_{1}(x, y)=\left(\begin{array}{c}
\tau_{L} x+y+\mu \\
-\delta_{L} x
\end{array}\right), \\
F_{2}(x, y)=\left(\begin{array}{c}
\tau_{R} x+y+\mu \\
-\delta_{R} x
\end{array}\right), \quad(x, y) \in \mathbb{R}^{2} .
\end{gathered}
$$

In this representation, the phase plane is divided into two regions $L=\{(x, y): x \leqslant 0, y \in \mathbb{R}\}$ and $R=\{(x, y): x>0, y \in \mathbb{R}\}$. $\tau_{L}$ and $\delta_{L}$ denote the trace and the determinant, respectively, of the Jacobian matrix $J_{L}$ in the half-plane $L$, and $\tau_{R}$ and $\delta_{R}$ are the trace and determinant of the Jacobian matrix $J_{R}$ in the region $R$. It is clear that

$$
J_{L}=\left(\begin{array}{cc}
\tau_{L} & 1 \\
-\delta_{L} & 0
\end{array}\right), \quad J_{R}=\left(\begin{array}{cc}
\tau_{R} & 1 \\
-\delta_{R} & 0
\end{array}\right) .
$$

The stability of the fixed point for the map (1) is determined by the eigenvalues of the corresponding Jacobian matrix $\lambda_{1,2}=\frac{1}{2}\left(\tau \pm \sqrt{\tau^{2}-4 \delta}\right)$. These fixed points at the two sides are given by

$$
\left(\frac{\mu}{\chi_{L}(1)},-\frac{\mu \delta_{L}}{\chi_{L}(1)}\right), \quad\left(\frac{\mu}{\chi_{R}(1)}-\frac{\mu \delta_{R}}{\chi_{R}(1)}\right),
$$

with $\chi(1)$ representing the value of the characteristic polynomial $\chi(\lambda)=\lambda^{2}-\tau \lambda+\delta$ for $\lambda=1$ in the considered half-plane.

As the parameter $\mu$ of the map (1) is varied from negative to positive values, the fixed point of (1) moves from $L$ to $R$ and a border-collision occurs at $\mu=0$. Let us choose the parameters such that $\delta_{L}<1$ and $\delta_{R}>1$. The conditions

$$
\begin{gathered}
\chi_{L}(1) \chi_{R}(1)>0, \\
-1-\delta_{L}<\tau_{L}<1+\delta_{L}, \quad-2 \sqrt{\delta_{R}}<\tau_{R}<2 \sqrt{\delta_{R}},
\end{gathered}
$$

then ensure that the fixed point is attracting for $\mu<0$ and a spiral repeller for $\mu>0$.

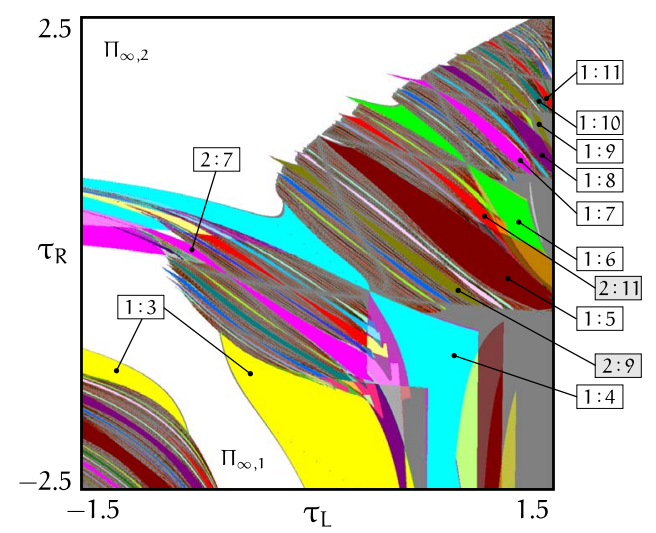

FIG. 1. (Color online) Chart of dynamical modes in the parameter plane $\left(\tau_{L}, \tau_{R}\right)$ with the remaining parameters fixed at $\delta_{L}=0.5$, $\delta_{R}=1.6$, and $\mu=0.05$.

In the present analysis we have assumed the following values for the determinants: $\delta_{L}=0.5$ and $\delta_{R}=1.6$. For $\mu<0$ the map (1) then has a single nontrivial stable fixed point with a negative $x$ coordinate. When $\mu$ changes sign, the $x$ coordinate of the fixed point also changes sign and the fixed point abruptly loses stability as a pair of complex-conjugate eigenvalues of the Jacobian matrix jump from the inside to the outside of the unit circle; i.e., the stable focus transforms abruptly into an unstable focus. If the parameters $\tau_{L}$ and $\tau_{R}$ of the map (1) are varied within the range delineated by (2), one can observe a large variety of dynamical phenomena associated with the interplay between homoclinic bifurcations and different forms of border-collision bifurcations [19]. Figure 1 shows the chart of dynamical modes (twoparameter bifurcation diagram) in the parameter plane $\left(\tau_{L}, \tau_{R}\right)$ for positive values of $\mu$. Inspection of this chart reveals the presence of a dense set of periodic tongues. The main resonance tongues are marked with the corresponding rotation numbers. In the 1:4 tongue, for instance, the system retraces itself after four iterations of the map (1). Between the tongues there are parameter combinations that lead to quasiperiodicity and chaos. The white regions $\Pi_{\infty, 1}$ and $\Pi_{\infty, 2}$ are domains where the trajectories of the map diverge to infinity for all initial conditions.

Depending on the parameter values, we observe a variety of different scenarios.

(i) If the values of the parameters $\tau_{L}$ and $\tau_{R}$ are chosen within a tongue of periodicity, then an attracting closed invariant curve softly arises from the fixed point as the parameter $\mu$ crosses the bifurcation point at $\mu=0$. This invariant curve is formed by the unstable manifolds of a saddle cycle and the points of the corresponding saddle and stable cycles. Figure 2 displays the bifurcation diagram (a) and the phase portrait (b) for a point $\left(\tau_{L}, \tau_{R}\right)$ in the 1:4 tongue.

(ii) If we choose the parameters $\tau_{L}$ and $\tau_{R}$ in a region of quasiperiodicity, the stable fixed point for $\mu<0$ turns into an unstable focus point on the $R$ side and quasiperiodic behavior arises. Figure 3 presents the bifurcation diagram for such a transition.

(iii) If $\tau_{L}$ or $\tau_{R}$ are varied within the region (2) for positive values of $\mu$, more complicated bifurcation phenomena are 

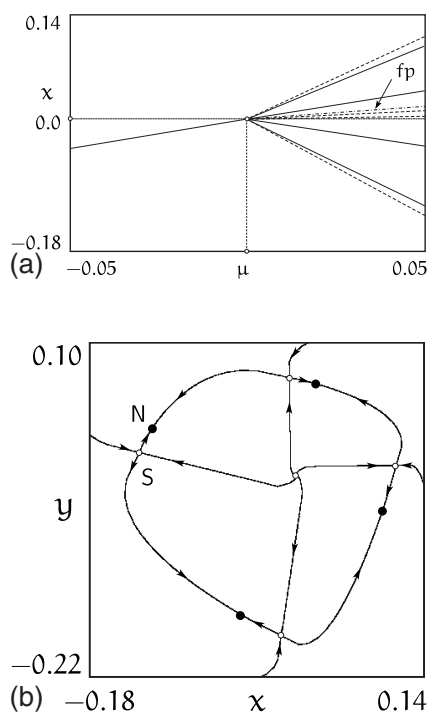

FIG. 2. Birth of a stable closed invariant curve from a stable fixed point in a border-collision bifurcation. $\tau_{L}=0.25$ and $\tau_{R}$ $=-0.25$. (a) Bifurcation diagram as the parameter $\mu$ varies from -0.05 to 0.05 . Solid lines to the right in the diagram mark the points of the stable period-4 cycle and dashed lines show the period-4 saddle. fp is the unstable fixed point. (b) Phase portrait for $\mu$ $=0.05$. The closed invariant curve is the union of the unstable manifolds of the saddle cycle and the points of the stable cycle. Points of stable cycles are marked with solid circles and points of saddle cycles with open circles.

possible in the transition from phase-locked dynamics to quasiperiodicity and vice versa. In particular, these phenomena include the border-collision fold bifurcation that is connected with the transitions from periodic to quasiperiodic dynamics and a modified variant of the multiple-attractor bifurcation in which a quasiperiodic attractor (or a modelocked periodic orbit) arises together with one (or more) stable cycles [23].

\section{TRANSITIONS FROM PHASE-LOCKED DYNAMICS TO CHAOS}

In each resonance tongue with the rotation number $r: q$ (see Fig. 1) the map displays an attracting closed invariant curve which typically takes the form of a saddle-node connection [Fig. 2(b)]. The unstable manifold of the period- $q$

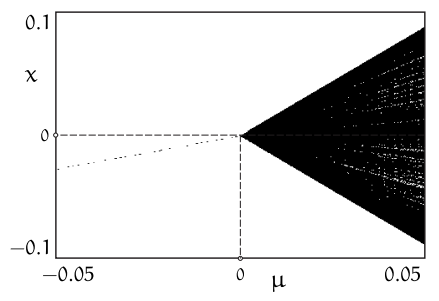

FIG. 3. Bifurcation diagram as the parameter $\mu$ varies from -0.05 to 0.05 . The diagram shows a direct transition from a stable period-1 focus cycle to a quasiperiodic orbit. The other parameters are $\tau_{L}=0.14, \tau_{R}=-0.25, \delta_{L}=0.5$, and $\delta_{R}=1.6$. saddle connects to the period- $q$ node, thus forming a closed attracting curve. For other parameter values, the closed invariant curve may be associated with a pair of saddle and focus cycles of similar periodicity [26].

In a couple of recent papers $[19,28]$ we have demonstrated that under variation of the parameters, this closed invariant curve is destroyed through a homoclinic bifurcation. However, the stable and saddle cycles may continue to exist after the torus destruction. With further change of the parameters, these cycles then merge and disappear in a border-collision fold bifurcation. As a result, between the curves of homoclinic bifurcation and of border-collision fold bifurcation there is a region of multistability, on the boundaries of which one can observe transitions with hysteresis. Using a dc-dc power converter as an example of a piecewisesmooth system, we have shown experimentally that the hysteretic transitions observed for the piecewise-linear normalform map actually occur in practical systems [19].

In the present paper we are interested in mechanisms of torus breakdown that relate to the transition from resonance behavior to chaotic dynamics. With this purpose we shall follow the bifurcations that take place as we leave the 1:4 resonance tongue of our normal-form map along three different directions in parameter space.

It is well known that the resonance tongues in piecewisesmooth systems are bounded by border-collision fold bifurcation curves $[2,15,28]$. As illustrated in Fig. 1, the 1:4 resonance tongue consists of two different parts separated by a so-called shrinking point in which the border-collision curves intersect. To the left of this point, two points of the $1: 4$ cycle fall in the negative half-plane $(x<0)$, as illustrated by the phase plot of Fig. 2(b), and in the lower right part of the tongue, three points of the cycle fall in the negative halfplane.

When the system leaves the resonance tongue through the border-collision fold bifurcation boundary $N_{+}^{C}$ of the upper part, one observes a transition from a resonance to ergodic torus. This transition is always followed by the breakdown of the torus through a homoclinic bifurcation. The transition from phase-locked periodic motion to chaos, which is the focus of the present study, only takes place on the boundaries of the lower right part of the resonance tongue.

Figure 4 shows a close-up of the chart of dynamical modes emphasizing this part of the 1:4 tongue. Here, the two border-collision curves are indicated by $N_{+}^{C} .1$ and 2 are homoclinic bifurcation curves, $N_{-}$is a smooth period-doubling bifurcation curve, and $N_{\text {div }}$ delineates the boundary of divergent behavior. The arrows marked $A, B$, and $C$ represent the directions in which we shall study the transitions in detail.

\section{PERIOD-DOUBLING ROUTE}

Let us first analyze what happens when moving from the inside to the outside of the resonance tongue through the period-doubling bifurcation curve $N_{-}$along the direction $A$. Results of a bifurcation analysis for the section $\left\{\left(\tau_{L}, \tau_{R}\right): 0.95 \leqslant \tau_{L} \leqslant 1.1, \tau_{R}=-1\right\}$ are presented in Figs. 5 and 6.

Figure 5(a) shows the bifurcation diagram obtained through direct simulation, and Fig. 5(b) displays the corre- 


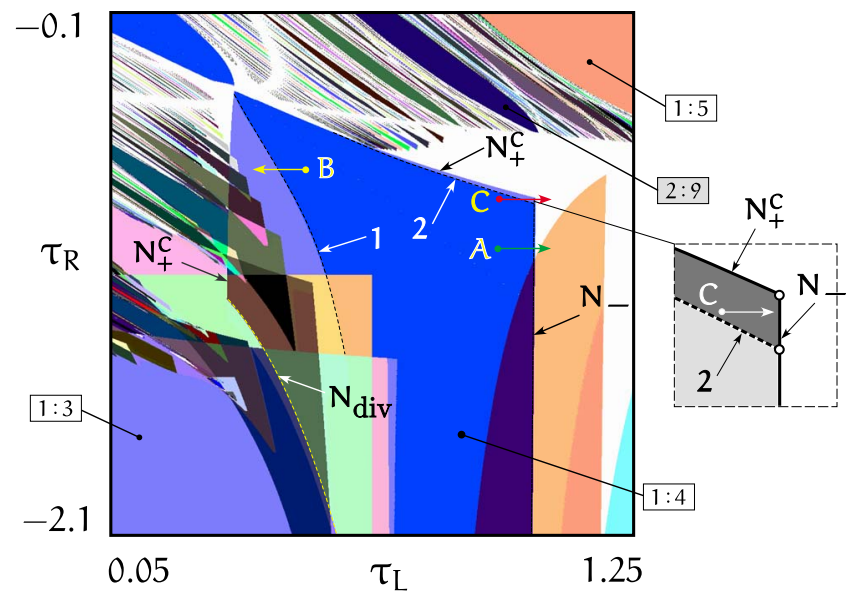

FIG. 4. (Color online) Part of the chart of the dynamical modes near the 1:4 resonance tongue. 1 and 2 are homoclinic bifurcation curves, $N_{+}^{C}$ denotes the border-collision fold bifurcation curves, and $N_{-}$is a smooth period-doubling bifurcation curve. The present analysis is concerned with the bifurcations that occur as we leave the 1:4 tongue along the directions $A, B$, and $C$, respectively.

sponding diagram as obtained by following the periodic orbits. To better illustrate the latter transition, a magnified section of Fig. 5(b) is presented in Fig. 6(a). The variation of the multipliers for the period-4 cycle for this section is shown in Fig. 6(b). At the point $\tau_{L}=\tau_{L}^{*} \approx 1.0236$ the largest multiplier of the period-4 cycle (in absolute value) crosses the unit circle through -1 and the period- 4 cycle turns into an unstable node [Fig. 6(b)]. Inspection of Figs. 5 and 6 shows that the loss of stability for the period-4 cycle is accompanied by the abrupt appearance of an eight-band strange attractor. It should be noted that this attractor contains a family
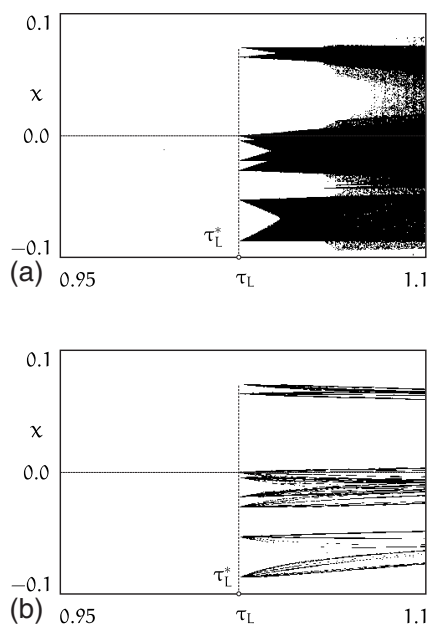

FIG. 5. Birth of an eight-band strange attractor through a smooth period doubling coinciding with a border-collision bifurcation. (a) One-parameter bifurcation diagram for the section along the direction $A$ in Fig. 4, $\tau_{R}=-1.0$. (b) Birth of a family of unstable cycles with periods that are multiples of 4 . The cycles arise through the border-collusion bifurcation at the point $\tau_{L}^{*} \approx 1.0236$. This diagram contains the unstable cycles with the periods $4,8,16,24,32$, and 40 .
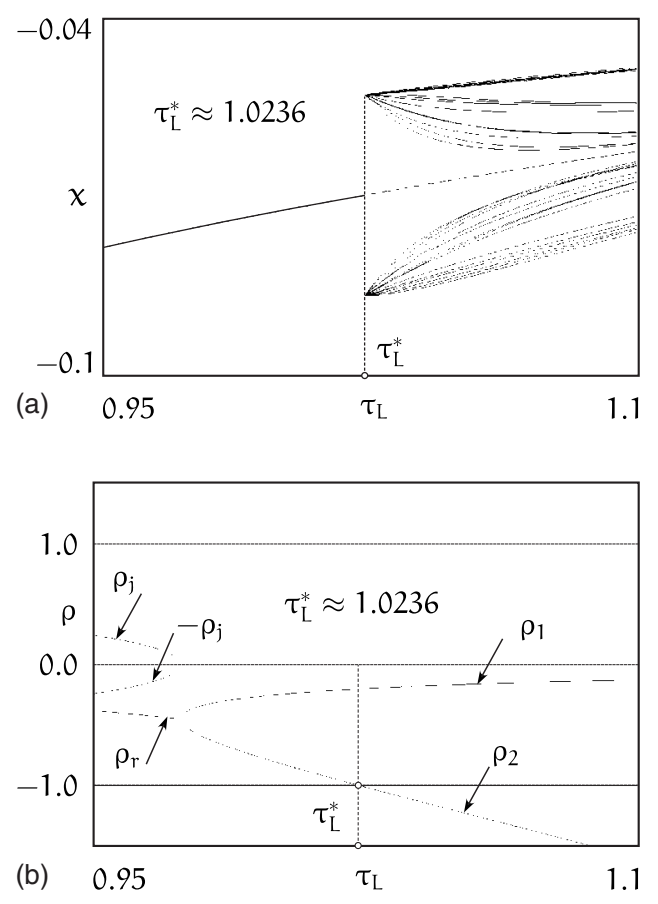

FIG. 6. (a) Magnified part of the bifurcation diagram presented in Fig. 5(b) showing the evolution of one branch. (b) Multiplier diagram illustrating the smooth period doubling of the period4 cycle. At the point $\tau_{L}^{*} \approx 1.0236$ the largest multiplier (in absolute value) of the period- 4 cycle crosses through -1 . The loss of stability of the period- 4 cycle is not accompanied by the appearance of a stable period- 8 cycle. Rather a whole family of unstable cycles arise through the border-collision bifurcation at $\tau_{L}^{*}$ [see Figs. 5(b) and $6(\mathrm{a})]$.

of unstable periodic orbits with periods that are multiples of 4. These cycles arise through the border-collision bifurcation at the point $\tau_{L}=\tau_{L}^{*}$ [see Figs. 5(b) and 6].

The bifurcation diagrams in Fig. 5 also show that at the point of period doubling, one of the points of the unstable period- 8 orbit hits the border $x=0$. This results in a direct transition to a chaotic attractor with eight bands. With further increase of the value of parameter $\tau_{L}$, the eight bands of the chaotic attractor merge first into a four-band chaotic attractor and subsequently into a single chaotic band. This transition is similar in its appearance to the transition observed by Maistrenko et al. [29] for the skew tent map. However, in our case the transition involves the destruction of a torus for the twodimensional map.

\section{A. Repeated folding of the stable and unstable manifolds}

Let us consider the characteristics of the bifurcational behavior shown in Figs. 5 and 6 in more detail in order to understand the mechanism of the transition between mode locking and chaos. Before the transition, the system displays a closed invariant curve that is the union of the unstable manifold of the period- 4 saddle cycle and the points of the stable-focus period-4 cycle. Inspection of Fig. 7 shows that the unstable manifold of the saddle fixed point has an infinite number of the linear pieces that fold at specific corner points before converging on the stable fixed points. 

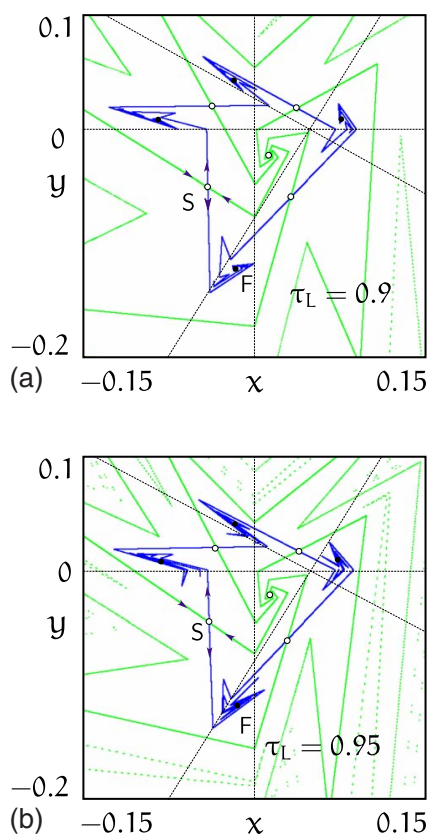

FIG. 7. (Color online) Nonsmooth torus for different values of $\tau_{L}$ : (a) $\tau_{L}=0.9$ and (b) $\tau_{L}=0.95$. This torus is the union of the unstable manifold of the saddle period- 4 cycle with the points of the stable-focus period- 4 cycle.

As shown by Banerjee and Grebogi [10], the unstable manifolds of the map (1) fold at every intersection with the $x$ axis and the image of a fold point is also a fold point. The stable manifolds fold at every intersection with the $y$ axis, and every preimage of a fold point is also a fold point. This creates the succession of folds in the invariant manifolds seen in Fig. 7.

Following [30-32] we denote the borderline as

$$
L C_{-1}:\{(x, y): x=0, y \in \mathbb{R}\} .
$$

The image of this line under one iteration of the map $F$, relation (1), is called the fold line $L C_{0}$ [32]:

$$
L C_{0}=F\left(L C_{-1}\right)=\{(x, y): y=0\} .
$$

The image of $L C_{0}$ is also a fold line:

$$
L C_{1}=F\left(L C_{0}\right)=\left\{(x, y): y=-\frac{\delta}{\tau}(x-\mu)\right\} .
$$

There will be two lines denoted by $L C_{1}$, one obtained by using the trace and determinant for the left half (region $L$ ) and the other obtained by using these parameters for the right half (region $R$ ).

To illustrate the role of these intersections, Fig. 8 shows the phase portrait of the map for $\tau_{R}=-0.98$ and $\tau_{L}=0.86$ where we have drawn the lines $L C_{-1}, L C_{0}$, and $L C_{1}$. It is evident from this figure that the unstable manifolds between the saddle points 1 and 2 cross the borderline $L C_{-1}$ at five points $A_{-1}^{+}$and are folded on the fold line $L C_{0}$, producing five corner points $A_{0}^{+}$. The forward images of these points are again corner points. The images of the points $A_{0}^{+}$lie on the line $L C_{1}$ and are denoted as $A_{1}^{+}$. Analogically, one segment of

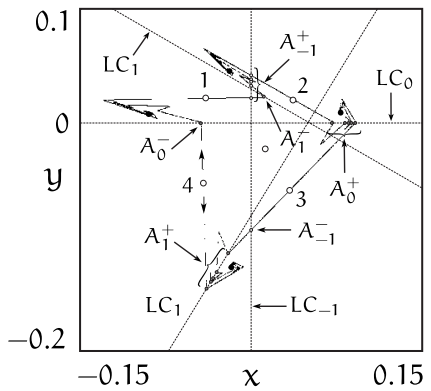

FIG. 8. Formation of the folds along the unstable manifold of a saddle period- 4 cycle, $\tau_{R}=-0.98, \tau_{L}=0.86 . L C_{-1}, L C_{0}$, and $L C_{1}$ are the critical lines. $1,2,3$, and 4 are the points of the saddle period4 cycle. $A_{-1}^{+}$and $A_{-1}^{-}$are the sets of the points of intersection between the border line $L C_{-1}$ and the arcs of the unstable manifold of the saddle cycle. $A_{0}^{+}$and $A_{0}^{-}$are the images of the $A_{-1}^{+}$and $A_{-1}^{-}$on the critical line $L C_{0}$. The arcs of the unstable manifold cross the line $L C_{-1}$ and fold on the $L C_{0}$ at the corner points $A_{0}^{+}$and $A_{0}^{-}$. $A_{1}^{+}$and $A_{1}^{-}$ are the images of the sets $A_{0}^{+}$and $A_{0}^{-}$on the line $L C_{1}$, respectively.

the unstable manifold between the points 3 and 4 of the saddle cycle crosses the line $L C_{-1}$ at the point $A_{-1}^{-}$and then folds on $L C_{0}$, producing the corner point $A_{0}^{-}$. The image of this point on the line $L C_{1}$ is denoted as $A_{1}^{-}$in Fig. 8.

\section{B. Period-doubling border-collision route}

Based on this structure of the stable and unstable manifolds, the main stages of the torus transformation can be understood. As the trace $\tau_{L}$ increases, at the point $\tau_{L} \approx 0.99$ the first homoclinic bifurcation occurs (or homoclinic contact by analogy with the homoclinic tangency in smooth maps) [see Fig. 9(a)]. With further increase in the value of $\tau_{L}$, the stable and unstable manifolds of the period- 4 saddle cycle intersect transversally to form a homoclinic structure [see Fig. 9(b)]. The intersection of the two manifolds implies the existence of a Smale horseshoe and, therefore, of an infinite number of high-periodic orbits [26]. After the homoclinic tangency, the attractor of the map is still the period-4 node, but the torus no longer exists.

In the meantime the multipliers of the stable period4 cycle change as shown in Fig. 10. At $\tau_{L} \approx 0.9744$, the complex-conjugate multipliers merge on the negative real line and the fixed point becomes a flip attractor. Following this, the two negative real eigenvalues move away from each other, and at $\tau_{L} \approx 1.0236$ one of the eigenvalues reach the unit circle on the negative real line. This marks a smooth period-doubling bifurcation, and it is known that beyond this point the torus cannot exist.

Since the map is linear on each side of the border at $x$ $=0$, the period- 8 cycle produced in the period doubling instantly moves away and one of its points collides with the border. This leads to the abrupt transition to an eight-band strange attractor.

Figure 11(a) shows the phase portrait after the perioddoubling bifurcation. The phase portrait contains the points of the unstable cycles with the periods $4,8,12,16,24,32$, and 40. As mentioned above, these cycles arise through a border-collision bifurcation occurring at the same parameter 

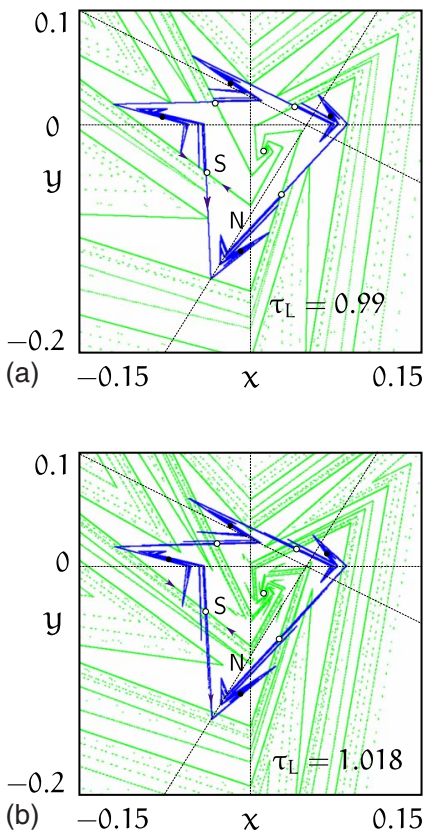

FIG. 9. (Color online) Torus destruction through homoclinic bifurcation before the smooth period doubling. (a) Phase portrait of the map near the first homoclinic contact (the analog of a homoclinic tangency in smooth maps), $\tau_{L}=0.99$. (b) Homoclinic intersections of the unstable and stable manifolds of the saddle period- 4 cycle, $\tau_{L}=1.018$. The multipliers of the stable period4 cycle are real and negative.

value as the period doubling, $\tau_{L}=\tau_{L}^{*}$. Figure 11(b) illustrates the phase portrait of the map for the single-band strange attractor.

It is possible that the period doubling takes place after a second homoclinic intersection. When that happens, we observe a hard transition to chaos through the period-doubling bifurcation. This transition is observed when the system leaves the resonance tongue along the section that crosses the line of the second homoclinic tangency 2 and the curve of the period doubling bifurcation $N_{-}$. The bifurcation diagram in Fig. 12 illustrates such a transition when we change the parameter $\tau_{L}$ along the direction $C$ (Fig. 4). To better illustrate the bifurcation transitions we do not include all the branches of the bifurcation diagram, but only a magnified view of one of the four.

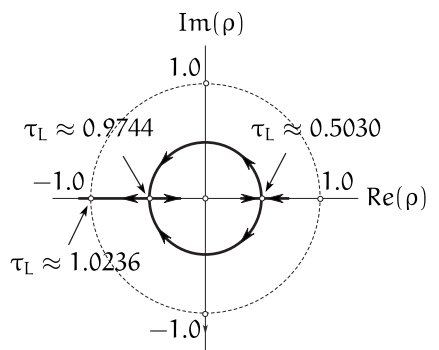

FIG. 10. Locus of the multipliers of the stable period-4 cycle. For $0.9744>\tau_{L}>0.5030$, the two multipliers are complex conjugated. At $\tau_{L}=1.0236$, the period- 4 cycle undergoes a period doubling.
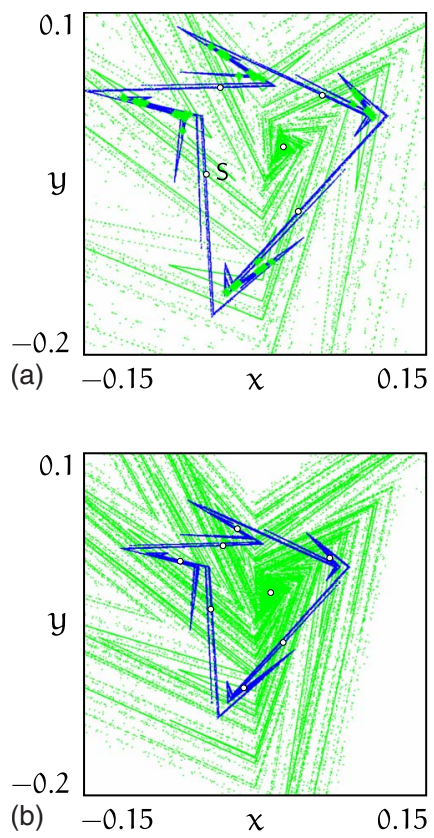

FIG. 11. (Color online) Phase portrait of the map after a perioddoubling bifurcation. (a) Eight-band strange attractor for $\tau_{L}$ $=1.035$. On the phase portrait one can see the points of the unstable cycles with the periods $4,8,12,16,24,32$, and 40 (see also the bifurcation diagram in Fig. 5). (b) Single-band strange attractor for $\tau_{L}=1.0725$.

First, the torus is destroyed through the homoclinic bifurcation. With further increase of $\tau_{L}$ the second homoclinic tangency occurs.

Between the point of the second homoclinic bifurcation $\tau_{L}^{H}$ and the point $\tau_{L}^{*}$ of the period-doubling bifurcation, the stable period-4 cycle coexists with the single-band strange attractor. At the point $\tau_{L}^{*}$ we can observe the abrupt transition to an eight-band strange attractor through the perioddoubling bifurcation.

As the parameter $\tau_{L}$ increases from the value $\tau_{L}^{*}$ the eightband strange attractor merges with the saddle period-4 cycle and disappears in a border-collision bifurcation. After this point, only the single strange attractor exists. In the region $\tau_{L}^{*}<\tau_{L}<\tau_{L}^{M}$ the eight-band and single-band strange attractors coexist as shown in Fig. 13. On the boundary of the second homoclinic tangency hard transition occurs from the single-band strange attractor to a stable period-4 cycle.

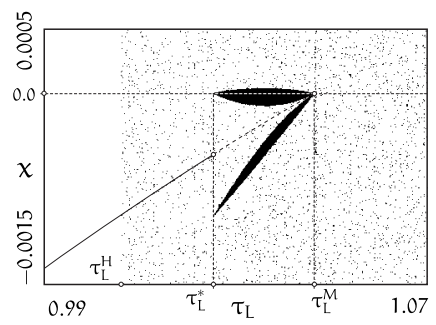

FIG. 12. Hard transition to chaos through period-doubling and homoclinic bifurcations, $\tau_{R_{*}}=-0.82$. $\tau_{L}^{H}$ is the point of the second homoclinic contact, and $\tau_{L}^{*}$ is the point of the period-doubling bifurcation. 


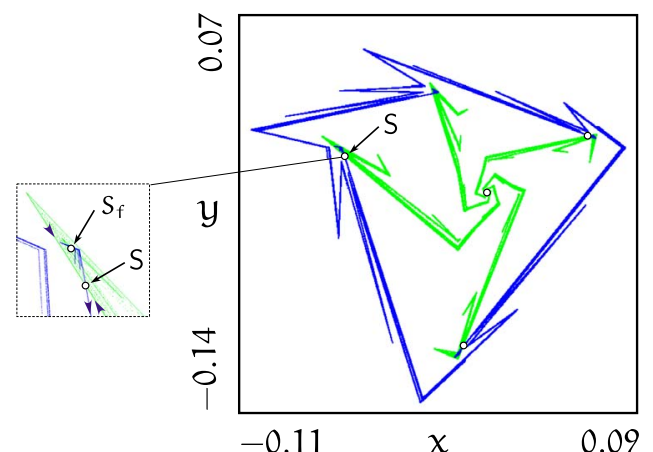

FIG. 13. (Color online) Phase portrait of the map for $\tau_{R}$ $=-0.82$ and $\tau_{L}=1.029$ in the region $\tau_{L}^{*}<\tau_{L}<\tau_{L}^{M}$ where the eightband and single-band strange attractors coexist. $S_{\mathrm{f}}$ is a saddle periodic point with two negative multipliers.

\section{HARD TRANSITION TO CHAOS THROUGH HOMOCLINIC INTERSECTION}

Now let us discuss the bifurcational behavior when we leave the resonance tongue along the direction $B$ (see Fig. 4). Figure 14 shows the bifurcation diagram for the section $\left\{\left(\tau_{L}, \tau_{R}\right): 0.4 \leqslant \tau_{L} \leqslant 0.45, \tau_{R}=-0.7\right\}$, and Fig. 15 illustrates the main stages of the torus transformation when changing the parameter $\tau_{L}$. We start from a point where the torus is formed by a saddle-focus connection.

Figure 15(a) displays the phase portrait of the map (1) near the "first" homoclinic contact. With further variation of $\tau_{L}$, the stable and unstable manifolds of the period- 4 saddle cycle intersect transversally and the closed invariant torus no longer exists [Fig. 15(b)]. At this stage, the stable focus cycle turns into stable node (the complex multipliers of the stable cycle become real and positive). Figure 15(c) shows the phase portrait after the second homoclinic contact. The boundary of the second homoclinic bifurcation is schematically shown by the dashed line in Fig. 4 and denoted as 1 . Here the stable period- 4 cycle coexists with the strange attractor.

As the parameter $\tau_{L}$ decreases the stable node period4 cycle merges with the saddle period- 4 cycle and disappears in a border-collision fold bifurcation. The domain between the lines of homoclinic bifurcation 1 and of bordercollision fold bifurcation $N_{+}^{C}$ is a region of multistability where the stable period-4 orbit coexists with strange and high-periodic attractors (Fig. 4). When crossing the boundaries of the upper part of the region multistability the system

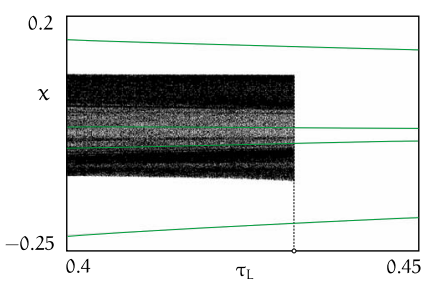

FIG. 14. (Color online) Bifurcation diagram illustrating the transition to chaos through the homoclinic bifurcation $0.4<\tau_{L}$ $<0.45, \tau_{R}=-0.7$.
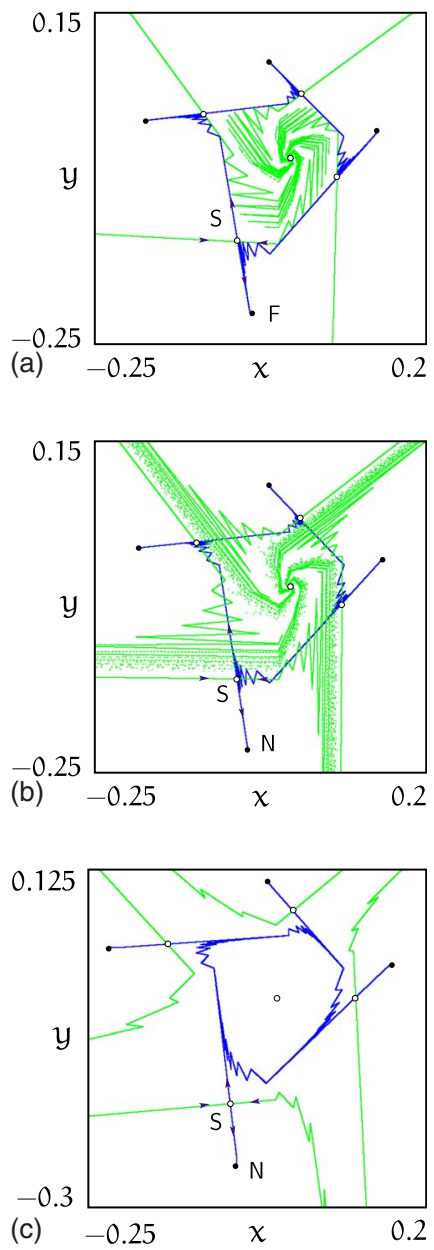

FIG. 15. (Color online) Main stages of torus destruction through a homoclinic bifurcation when the system leaves the resonance tongue through the left boundary (the homoclinic bifurcation curve is denoted as 1). $\tau_{R}=-0.7$. (a) Phase portrait of the map near the first homoclinic contact (the analogue of a homoclinic tangency in the smooth maps), $\tau_{L}=0.457$. (b) Homoclinic intersections of the unstable and stable manifolds of the saddle period-4 cycle, $\tau_{L}$ $=0.44$. (c) Phase portrait of the map after the torus is destroyed. Here the stable node period- 4 cycle $N$ coexists with the strange attractor. The basins of attraction of periodic and strange attractors are separated by the stable manifold of the period- 4 saddle cycle $S$. $\tau_{L}=0.4$.

displays hysteretic transitions from the periodic to the strange attractor and vice versa.

On the boundaries of the bottom part of this region, the dynamical behavior is more complicated, as tongues of various periodicity intersect with the 1:2 tongue. Here one can observe a hard transition from period-4 cycle to a strange or high-periodic attractor and vice versa.

Between the lines 2 and $N_{+}^{C}$ we find a region of bistability where the stable period- 4 cycle coexists with the strange attractor. As a result, along the whole boundary of this region we may observe hysteretic transitions. Figure 16 displays a bifurcation diagram illustrating the hysteretic phenomena near the right boundary of the 1:4 tongue. As the parameter $\tau_{L}$ increases, the stable focus and saddle period- 4 orbits collide and disappear in a border-collision fold bifurcation at the 


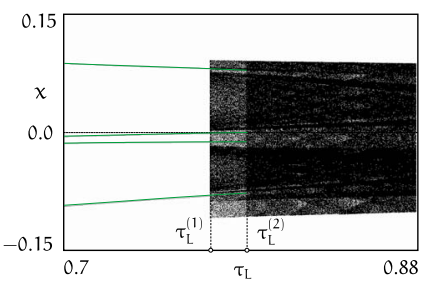

FIG. 16. (Color online) Bifurcation diagram illustrating the hysteretic phenomena on the right boundary of the 1:4 tongue, $\tau_{R}$ $=-0.7$.

point $\tau_{L}^{(2)} \cdot \tau_{L}^{(1)}$ is the point of homoclinic bifurcation. Between the points $\tau_{L}^{(1)}$ and $\tau_{L}^{(2)}$ the stable periodic orbit coexists with a strange attractor. We conclude that hard hysteretic transitions from one dynamical mode to another take place at the points $\tau_{L}^{(1)}$ and $\tau_{L}^{(2)}$. With increasing $\tau_{L}$, for example, a hard transition from a periodic to a strange attractor takes place at $\tau_{L}^{(2)}$.

\section{CONCLUSIONS}

Many systems of interest in physics, engineering, and other sciences display discontinuities that lead to a dynamical description in terms of piecewise-smooth maps. In such systems, quasiperiodic or phase-locked resonant behavior on the surface of a torus can arise through a special type of border-collision bifurcation in which a pair of complexconjugated multipliers for a stable periodic orbit jumps out of the unit circle. With further parameter variation, the torus may be destroyed through a number of different mechanisms, giving birth to chaos.

Afraimovich and Shilnikov have proposed three possible mechanisms for the transition from torus to chaos in smooth maps. In this paper we investigated three specific routes of torus destruction leading from phase-locked dynamics to chaos in piecewise-smooth maps. Using the appropriate piecewise-linear normal-form map as a tool, we showed that the routes to chaos in nonsmooth maps may display significant differences from the mechanisms described by Afraimovich and Shilnikov.

In one of the routes reported in this paper, a homoclinic intersection first destroys the torus. In the absence of the torus, the stable node undergoes a period doubling, immediately followed by a border collision that gives birth to the chaotic orbit. In another route, the first homoclinic tangency is followed by a second homoclinic tangency, which gives birth to a single-band strange attractor. But the stable periodic orbit persists. At a different parameter value, this periodic orbit undergoes a period-doubling bifurcation, again immediately followed by a border collision. This creates a different multiband chaotic orbit. If the orbit before period doubling was period $n$, the strange attractor has $2 n$ bands. The multiband attractor is destroyed at a border-collision fold bifurcation, where we see a hard transition from one chaotic orbit to another.

In the third route, the first homoclinic tangency is followed by a second homoclinic tangency and a strange attractor is born. This attractor coexists with the stable periodic orbit for some parameter interval. At a specific parameter value, the stable node (or focus) collides with the saddle on the border and both are destroyed through a border-collision fold bifurcation.

\section{ACKNOWLEDGMENTS}

The work was supported by the Russian Foundation for Basic Research (Grant No. 06-01-00811) and by the Danish Natural Science Foundation through the Center for Modelling, Nonlinear Dynamics, and Irreversible Thermodynamics (MIDIT). S. D. acknowledges support from the Council for Scientific and Industrial Research, India.
[1] Nonlinear Phenomena in Power Electronics, edited by S. Banerjee and G. C. Verghese (IEEE Press, New York, 2001).

[2] Zh. T. Zhusubaliyev and E. Mosekilde, Bifurcations and Chaos in Piecewise-Smooth Dynamical Systems (World Scientific, Singapore, 2003).

[3] B. Brogliato, Nonsmooth Mechanics-Models, Dynamics and Control (Springer Verlag, New York, 1999).

[4] J. Laugesen and E. Mosekilde, Comput. Oper. Res. 33, 464 (2006).

[5] M. I. Feigin, Prikl. Mat. Mekh. 34, 861 (1970).

[6] M. I. Feigin, Forced Oscillations in Systems with Discontinuous Nonlinearities (Nauka, Moscow, 1994) (in Russian).

[7] H. E. Nusse and J. A. Yorke, Physica D 57, 39 (1992).

[8] H. E. Nusse, E. Ott, and J. A. Yorke, Phys. Rev. E 49, 1073 (1994).

[9] M. di Bernardo, M. I. Feigin, S. J. Hogan, and M. E. Homer, Chaos, Solitons Fractals 10, 1881 (1999).

[10] S. Banerjee and C. Grebogi, Phys. Rev. E 59, 4052 (1999).
[11] S. Banerjee, M. S. Karthik, G. Yuan, and J. A. Yorke, IEEE Trans. Circuits Syst., I: Fundam. Theory Appl. 47, 389 (2000).

[12] S. Banerjee, P. Ranjan, and C. Grebogi, IEEE Trans. Circuits Syst., I: Fundam. Theory Appl. 47, 633 (2000).

[13] M. di Bernardo, C. J. Budd, and A. R. Champneys, Physica D 154, 171 (2001).

[14] Zh. T. Zhusubaliyev, E. A. Soukhoterin, and E. Mosekilde, Int. J. Bifurcation Chaos Appl. Sci. Eng. 11, 2977 (2001).

[15] Zh. T. Zhusubaliyev, E. A. Soukhoterin, and E. Mosekilde, Chaos, Solitons Fractals 13, 1889 (2002).

[16] M. di Bernardo, C. J. Budd, and A. R. Champneys, Physica D 160, 222 (2001).

[17] M. di Bernardo, P. Kowalczyk, and A. Nordmark, Physica D 170, 175 (2002).

[18] A. B. Nordmark, Nonlinearity 14, 1517 (2001).

[19] Zh. T. Zhusubaliyev, E. Mosekilde, S. M. Maity, S. Mohanan, and S. Banerjee, Chaos 16, 023122 (2006).

[20] Zh. T. Zhusubaliyev and E. Mosekilde, IEEE Trans. Circuits 
Syst., I: Fundam. Theory Appl. 53, 1839 (2006).

[21] Zh. T. Zhusubaliyev and E. Mosekilde, in Handbook of Chaos Control, edited by Schöll and Schuster (Wiley-VCH, Weinheim, 2007), pp. 755-775.

[22] S. Maity, D. Tripathy, T. K. Bhattacharya, and S. Banerjee, IEEE Trans. Circuits Syst., I: Fundam. Theory Appl. 54, 1120 (2007).

[23] Zh. T. Zhusubaliyev, E. Mosekilde, and S. Banerjee, Int. J. Bifurcation Chaos Appl. Sci. Eng. (to be published).

[24] V. S. Afraimovich and L. P. Shilnikov, Am. Math. Soc. Transl. 149, 201 (1991).

[25] D. G. Aronson, M. A. Chori, G. R. Hall, and R. P. McGhee, Commun. Math. Phys. 83, 303 (1982).

[26] Yu. A. Kuznetsov, Elements of Applied Bifurcation Theory (Springer-Verlag, New York, 2004).
[27] V. Maistrenko, Y. Maistrenko, and E. Mosekilde, Phys. Rev. E 67, 046215 (2003).

[28] Zh. T. Zhusubaliyev and E. Mosekilde, Phys. Lett. A 351, 167 (2006).

[29] Yu. L. Maistrenko, V. L. Maistrenko, S. I. Vikul, and L. O. Chua, Int. J. Bifurcation Chaos Appl. Sci. Eng. 5, 653 (1995).

[30] C. Mira, L. Gardini, A. Barugola, and J. C. Cathala, Chaotic Dynamics in Two-Dimensional Noninvertible Maps (World Scientic, Singapore, 1996).

[31] C. E. Frouzakis, L. Gardini, I. G. Kevrekidis, G. Millerioux, and C. Mira, Int. J. Bifurcation Chaos Appl. Sci. Eng. 7, 1167 (1997).

[32] I. Sushko and L. Gardini, in Business Cycle Dynamics: Models and Tools, edited by T. Puu and I. Sushko (Springer, Berlin, 2006), pp. 49-78. 